\title{
Neural Variability, Detection Thresholds, and Information Transmission in the Vestibular System
}

\author{
Soroush G. Sadeghi, ${ }^{1 \star}$ Maurice J. Chacron, ${ }^{1 \star}$ Michael C. Taylor, ${ }^{1,2}$ and Kathleen E. Cullen ${ }^{1}$ \\ ${ }^{1}$ Department of Physiology, McGill University, Montreal, Quebec, Canada H3G 1Y6, and 2Department of Physics, McGill University, Montreal, Quebec, \\ Canada H3G 1 Y6
}

\begin{abstract}
A fundamental issue in neural coding is the role of spike timing variation in information transmission of sensory stimuli. Vestibular afferents are particularly well suited to study this issue because they are classified as either regular or irregular based on resting discharge variability as well as morphology. Here, we compared the responses of each afferent class to sinusoidal and random head rotations using both information theoretic and gain measures. Information theoretic measures demonstrated that regular afferents transmitted, on average, two times more information than irregular afferents, despite having significantly lower gains. Moreover, consistent with information theoretic measures, regular afferents had angular velocity detection thresholds that were $50 \%$ lower than those of irregular afferents ( $\left.\sim 4 \mathrm{vs} 8^{\circ} / \mathrm{s}\right)$. Finally, to quantify the information carried by spike times, we added spike-timing jitter to the spike trains of both regular and irregular afferents. Our results showed that this significantly reduced information transmitted by regular afferents whereas it had little effect on irregular afferents. Thus, information is carried in the spike times of regular but not irregular afferents. Using a simple leaky integrate and fire model with a dynamic threshold, we show that differential levels of intrinsic noise can explain differences in the resting discharge, the responses to sensory stimuli, as well as the information carried by action potential timings of each afferent class. Our experimental and modeling results provide new insights as to how neural variability influences the strategy used by two different classes of sensory neurons to encode behaviorally relevant stimuli.
\end{abstract}

Key words: vestibular afferents; information theory; spike timing; regular afferents; detection threshold; heterogeneity

\section{Introduction}

The nervous system uses a variety of neural codes ranging from rate codes where spike timing appears unimportant (Adrian and Zotterman, 1926; Connor et al., 1990; Carr, 1993; Salinas et al., 2000; Johansson and Birznieks, 2004) to temporal codes where spike timing is critical for coincidence detection (Carr and Konishi, 1990; Carr, 1993; Joseph and Hyson, 1993), first spike latency (Johansson and Birznieks, 2004), or improving stimulusresponse correlations (Jones et al., 2004). This has sparked intense debate on whether variability in spike timing actually plays a role in sensory processing: whereas variability is averaged out to obtain the time dependent firing rate in a rate code, variability may carry information in a temporal code (Stein et al., 2005). Studies exploiting relatively simple systems with well described organization and natural stimuli are needed to make progress toward understanding the role of neural variability in sensory processing.

The vestibular system provides information about head motion relative to space that is necessary for maintaining posture,

\footnotetext{
Received Aug. 8, 2006; revised Nov. 25, 2006; accepted Dec. 14, 2006.

This work was supported by the Canadian Space Agency (K.E.C.) and the Canadian Institutes of Health Research (K.E.C., M.J.C). We thank R. Krahe, M. Beraneck, M. Van Horn, N. Sadeghi, A. Krishnaswamy, E. Cook, L. Maler, and S. Cherif for useful discussions.

*S.G.S. and M.J.C. contributed equally to this work.

Correspondence should be addressed to Kathleen E. Cullen, Aerospace Medical Research Unit, 3655 Drummond Street, Montreal, Quebec, Canada H3G 1Y6. E-mail: Kathleen.cullen@mcgill.ca.

DOI:10.1523/JNEUROSCI.4690-06.2007

Copyright $\odot 2007$ Society for Neuroscience $\quad$ 0270-6474/07/270771-11\$15.00/0
}

computing spatial orientation, and perceiving self-motion. This system is well characterized anatomically and encodes head velocity over the natural frequency range $(0-20 \mathrm{~Hz}$ ) (Grossman et al., 1988; Armand and Minor, 2001; Huterer and Cullen, 2002). Furthermore, vestibular afferents at the sensory periphery are particularly well suited for studying the role of neural variability because they display a wide range of resting discharge variability. Afferents in this system are characterized as either regular or irregular based on their resting discharge, which is correlated with distinct morphological features at their peripheral terminations (Baird et al., 1988; Fernandez, 1988; Goldberg, 2000). Afferent responses to sensory input have been traditionally characterized by estimating the gain and phase over several cycles of sinusoidal head rotation (Goldberg and Fernandez, 1971b; Goldberg, 2000; Hullar et al., 2005). A limitation of this approach is that it averages response variability.

Here, we use information theoretic and signal detection analysis to study the role of neural variability in determining the responses of each afferent class to random as well as $0-20 \mathrm{~Hz}$ sinusoidal head rotations in primates. Our results using information theory reveal that, whereas irregular afferents best code for high frequencies, regular afferents instead transmit information about the detailed time course of the stimulus because of their lower variability. Consistent with these findings, we found that regular afferents had lower velocity detection thresholds than irregular afferents. Also, regular afferents are significantly more sensitive to addition of spike-timing jitter than irregular affer- 
ents. This demonstrates the existence of a temporal code at the peripheral level in the vestibular system.

Finally, we used a simple leaky integrate-and-fire model with a dynamic threshold to mimic the response properties of regular and irregular vestibular afferents. Our model explains the role of intrinsic noise in determining variability, information transmission, and detection thresholds, as well as the sensitivity to spiketiming jitter of each afferent class. Together, our experimental and theoretical findings establish that neural variability can play an important role in determining the neural code used by mammalian sensory systems.

\section{Materials and Methods}

Surgical preparations. Two macaque monkeys (Macaca fascicularis) were prepared for chronic extracellular recording using aseptic surgical techniques described previously (Sylvestre and Cullen, 1999). Briefly, the animals were preanesthetized using ketamine hydrochloride (12-15 mg/ $\mathrm{kg}$, i.m.). In addition, atropine sulfate $(0.04 \mathrm{mg} / \mathrm{kg}$, i.m.) and valium (1 $\mathrm{mg} / \mathrm{kg}$, i.m.) were administered to reduce salivation and provide muscle relaxation, respectively. Surgical levels of anesthesia were induced using $2-3 \%$ isoflurane gas, inhaled through an endotracheal tube, and were then maintained at $0.8-1.5 \%$. During the surgery, a dental acrylic implant was attached to each animal's skull using stainless-steel screws. Within the implant were embedded a stainless-steel post that was used to restrain the animal's head during the experiment, and two stainless-steel recording chambers, which were positioned stereotaxically on the skull to allow recording from the vestibular nerve where it emerges from the internal auditory meatus. After the surgery, the animals were administered buprenorphine $(0.01 \mathrm{mg} / \mathrm{kg}$, i.m.) for postoperative analgesia, and the antibiotic cephazolin (Ancef; $25 \mathrm{mg} / \mathrm{kg}$, i.m., for $5 \mathrm{~d}$ ). Animals were given at least 2 weeks to recover from the surgery before experiments began. All procedures were approved by the McGill University Animal Care Committee and were in compliance with the guidelines of the Canadian Council on Animal Care.

Data acquisition. The experimental setup, apparatus, and methods of data acquisition have been described in detail previously (Cullen and Minor, 2002; Huterer and Cullen, 2002; Sadeghi et al., 2006). During experimental sessions, the monkey was comfortably seated in a primate chair mounted on a vestibular turntable. Eye and head movements were recorded using the magnetic search coil technique, collected by the QNX-based real-time data acquisition system REX, sampled at $1 \mathrm{kHz}$, low-pass filtered at $250 \mathrm{~Hz}$ (analog eight-pole Bessel filter), and stored to digital audiotape for subsequent playback. Extracellular single-unit activity was recorded with glass microelectrodes (24-27 M $\Omega$ ), the depth of which was controlled using a precision hydraulic microdrive (Narishige, Tokyo, Japan). The vestibular nerve was approached through the floccular lobe of the cerebellum, as identified by its eye-movement-related activity (Lisberger and Pavelko, 1986; Cullen and Minor, 2002); entry to the nerve was preceded by a silence, indicating that the electrode had left the cerebellum. Action potentials from extracellular recordings were discriminated using an oscilloscope windowing circuit (BAK Electronics, Mount Airy, MD); Schmitt trigger levels and window size were manually set and monitored throughout playback sessions to ensure no exclusion or double-counting of spikes. The resulting spike train was digitized at 1 $\mathrm{kHz}$. Spike train, eye, and head position signals were subsequently imported into Matlab (Mathworks, Natick, MA) and analyzed with custom-written algorithms.

Experimental design. We confirmed that each unit discharged in a manner consistent with previous characterizations of the afferents that innervate the horizontal semicircular canals (Goldberg, 2000). All afferents were activated by rotational head movements toward the side of the recording, but not in response to static tilts. In addition, we verified that responses were not modulated during saccadic or pursuit eye movements.

Each unit's spontaneous activity (i.e., discharge in the absence of head movement) was initially recorded for $2 \mathrm{~min}$ before vestibular stimulation so that the regularity of discharge could be computed. Afferents were then recorded from during passive whole-body sinusoidal $(1-4 \mathrm{~Hz}$, max- imum velocity $50 \%$ s) rotations about an earth vertical axis (yaw axis). Next, passive horizontal head on body rotations about the yaw axis were applied using a torque motor (Servostar S602; Kollmorgen AKM servomotor; Danaher Motion, Dusseldorf, Germany), which was securely coupled by precision universal joints to the post implanted on the monkey's head. We used two types of head velocity stimuli. The first type consisted of single frequency sinusoidal rotations at frequencies of 5-15 $\mathrm{Hz}$ (maximum velocity, $50 \%$ ). The second type consisted of broadband noise stimuli characterized by a Gaussian distribution of rotational velocities with zero mean and an SD of $20 \%$ s that was low-pass filtered at 30 $\mathrm{Hz}$. The acceleration profile of this stimulus had a Gaussian distribution with zero mean and an SD of $2000 \% \mathrm{~s}^{2}$. Spectral analysis showed that the power spectral estimate of the stimulus [computed using multitaper estimation techniques with eight Slepian functions (Jarvis and Mitra, 2001)] was constant for frequencies up to $20 \mathrm{~Hz}$ (data not shown) and the stimuli were low-passed using a 51st-order finite-response filter with a Hamming window and cutoff frequency set to $30 \mathrm{~Hz}$ (Huterer and Cullen, 2002).

Analysis of background activity. The firing characteristics of vestibular neurons were classified by analyzing the distribution of interspike intervals (ISIs) recorded during spontaneous background activity. The coefficient of variation $(\mathrm{CV})$ of a sequence of ISIs was calculated from the mean $\left(\mu_{\mathrm{ISI}}\right)$ and SD $\left(\sigma_{\mathrm{ISI}}\right)$ of the ISI distribution $\left(\mathrm{CV}=\sigma_{\mathrm{ISI}} / \mu_{\mathrm{ISI}}\right)$. Because $\mathrm{CV}$ varies with the mean ISI, we used a normalized measure $\mathrm{CV}^{*}$ to classify afferents (Goldberg et al., 1984). Neurons with a $\mathrm{CV}^{*}<0.15$ were classified as regular whereas those with a $\mathrm{CV}^{\star} \geq 0.15$ were classified as irregular (Haque et al., 2004). Results were pooled between high- and low-gain irregular afferents (Baird et al., 1988). Each spike train was converted into a binary sequence where a value of 1 was assigned to each action potential and the spike train power spectrum $P_{0}(f)$ of this binary sequence was computed using multitaper estimation techniques with eight Slepian functions (Jarvis and Mitra, 2001).

Afferent tuning. Responses of afferents to broadband $(0-20 \mathrm{~Hz})$ vestibular stimulation were characterized using two alternate measures. The coherence $C(f)$ between the head velocity signal $s(t)$ and the spike train $r(t)$ was calculated as a measure of the strength of the response to the stimulus at a frequency $f$, and is given by $C(f)=\left|P_{\mathrm{rs}}(f)\right|^{2} /\left[P_{\mathrm{ss}}(f) P_{\mathrm{rr}}(f)\right]$, where $P_{\mathrm{rs}}$ denotes the stimulus-response cross spectrum, $P_{\mathrm{ss}}$ is the power spectrum of the stimulus, and $P_{\mathrm{rr}}$ is the power spectrum of the spike train. A lower bound estimate of the mutual information rate density is obtained from the coherence by $I(f)=-\log _{2}[1-C(f)]$ (Rieke et al., 1996; Theunissen et al., 1996; Chacron et al., 2005b) and is measured in bits per second per Hertz. To account for the fact that the mutual information rate (MI) density increases with firing rate (Borst and Haag, 2001), we normalized it by the mean firing rate $f_{\mathrm{r}}$ (measured in spikes per second) during stimulation: $\mathrm{MI}(f)=I(f) / f_{\mathrm{r}}$. $\mathrm{MI}(f)$ is thus measured in bits per spike per Hertz. In addition, conventional transfer functions were calculated to determine the response gain, which is obtained by dividing the stimulus-response cross spectrum by the power spectrum of the stimulus: $G(f)=\left|P_{\text {sr }}(f) / P_{\text {ss }}(f)\right|$. For comparison, response gains were also computed from sinusoidal stimuli in the same manner.

To quantify the amount of information transmitted by vestibular afferents in different frequency ranges, we integrated the normalized information rate density $\mathrm{MI}(f)$ over both low $(0.5-5 \mathrm{~Hz})$ and high frequencies $(15-20 \mathrm{~Hz})$. For comparison, we also averaged the gain $G(f)$ over these same frequency ranges. Time-dependent firing rates of the afferents were represented using a spike density function in which a Gaussian function with an SD of 5 was convolved with the spike train. Spike-timing jitter was introduced by adding to each spike time a random amount drawn from a Gaussian distribution with zero mean and preset SD. The resulting spike train was then analyzed in the same manner as the raw ones. Error bars were estimated over 30 realizations of jitter.

Detection threshold calculation. Estimates of the time-dependent firing rate $f(t)$ during sinusoidal head rotation were obtained by low-pass filtering the spike train $r(t)$ using a Kaiser window with cutoff frequency greater than the stimulus frequency by $0.1 \mathrm{~Hz}$ (Oppenheim and Schafer, 1989). At each frequency of rotation, an afferent's dynamic lead time relative to head velocity was estimated by least-squares optimization of the equation: $f(t)=($ gain $) \times\left[s\left(t-t_{d}\right)\right]+$ bias, where $f(t)$ is firing rate, 
$s(t)$ is head velocity, gain is a constant value, $t_{\mathrm{d}}$ is the dynamic lead time (in milliseconds), and bias is an offset. A minimum of 10 cycles were included in the fit and the variance-accounted-for was computed as $1-$ $[\operatorname{var}($ est $-f) / \operatorname{var}(f)]$, where var represents variance, est represents the modeled firing rate, and $f$ represents the actual firing rate (Sylvestre and Cullen, 1999; Roy and Cullen, 2001). The firing rate was then plotted as a function of the shifted stimulus $s(t)$ to obtain the instantaneous firing rate-head velocity curve. We then computed the mean and variance of the firing rate distribution for each head velocity using a binwidth of $1 \%$. The degree of overlap between the firing rate distribution obtained for head velocity $I$ and the one obtained under resting discharge was quantified using the $\mathrm{d}^{\prime}$ measure from signal detection theory (Green and Swets, 1966):

$$
d^{\prime}(I)=\frac{|\mu(I)-\mu(0)|}{\sqrt{\left(\sigma^{2}(I)+\sigma^{2}(0)\right) / 2}},
$$

where $\mu(I)$ and $\sigma^{2}(I)$ are the mean and variance of the firing rate distribution at head velocity $I$, respectively. $\mu(0)$ and $\sigma^{2}(0)$ are the mean and variance of the firing-rate distribution under resting discharge, respectively. It was observed that $\mathrm{d}^{\prime}$ increased linearly as a function of $|I|$ and that the data could be fitted with a first-order polynomial. We thus used our linear fit to interpolate between data points to find the detection threshold $v_{T}$ as the lowest absolute head velocity satisfying $\mathrm{d}^{\prime}\left(v_{T}\right)>1$ (Snippe and Koenderink, 1992). We also used the firing rate distribution obtained for 0 head velocity to compute discrimination threshold and found that these did not differ significantly from the detection thresholds computed from the spontaneous activity (data not shown).

Stimulus reconstruction. Spike trains $r(t)$ recorded during broadband $(0-20 \mathrm{~Hz})$ vestibular stimulation were used to reconstruct a linear estimate of the stimulus $s(t)$. It is assumed that the time-dependent stimulus can be described by a first-order Voltera series (Rieke et al., 1996):

$$
s_{\text {est }}(t)=\int \mathrm{d} \tau \mathrm{K}(\tau) \mathrm{r}(\mathrm{t}-\tau) .
$$

The kernel $K(\tau)$ that minimizes the mean-square error $\varepsilon^{2}=<[s(t)-$ $\left.s_{\text {est }}(t)\right]^{2}>$ can be computed in the Fourier domain from the cross spectrum and power spectrum of the response as follows (Rieke et al., 1996; Dayan and Abbott, 2001):

$$
\tilde{K}(f)=\frac{P_{\mathrm{rs}}(-f)}{P_{\mathrm{rr}}(f)},
$$

where $\tilde{K}(f)$ is the Fourier transform of $K(t)$. We assessed the quality of linear stimulus estimation by computing the coding fraction as follows (Gabbiani, 1996; Rieke et al., 1996):

$$
\mathrm{CF}=1-\frac{\varepsilon}{\sigma_{\text {stim }}},
$$

where $\varepsilon$ is the root mean squared error and $\sigma_{\text {stim }}$ is the SD of the stimulus $s(t)$. The coding fraction ranges between 0 and 1 and represents the fraction of the stimulus correctly estimated.

Modeling. We used a modified leaky integrate-and-fire model with a dynamic threshold (Chacron et al., 2000, 2001, 2005b) to model vestibular afferents. The voltage $v$ and threshold $w$ obey the following differential equations over times between action potentials as follows:

$$
\begin{gathered}
\dot{v}=-\frac{v}{\tau_{v}}+\frac{I_{\text {synap }}}{\tau_{v}} \\
\dot{w}=\left(w_{0}-w\right) / \tau_{w},
\end{gathered}
$$

where $\tau_{v}$ is the membrane time constant, $\tau_{w}$ is the threshold recoverytime constant, and $w_{0}$ is the equilibrium value for the threshold. When $v(t)=w(t), v$ is reset to 0 whereas $w$ is incremented by $\Delta w$ and an action potential is said to have occurred at time $t$. $v$ is subsequently kept at 0 for the duration of the absolute refractory period $T_{\text {refrac }}$. Cumulative increases in threshold mimic cumulative afterhyperpolarization in the
Table 1. Parameter values used for the simulations

\begin{tabular}{lll}
\hline & Regular & Irregular \\
\hline$I_{\text {bias }}$ & 0.0515 & 0.049 \\
$\tau_{\mathrm{v}}(\mathrm{ms})$ & 1 & 1 \\
$\tau_{\mathrm{w}}(\mathrm{ms})$ & 9.5 & 9.5 \\
$\mathrm{~W}_{0}$ & 0.05 & 0.05 \\
$\Delta \mathrm{w}$ & 0.003 & 0.001 \\
$T_{\text {refrac }}(\mathrm{ms})$ & 1 & 1 \\
$\sigma$ & 0.00007 & 0.0015 \\
$G_{H}(\mathrm{~ms} / \mathrm{deg})$ & $0 / 0.0156$ & $0 / 0.0315$ \\
$G_{\mathrm{A}}(\mathrm{ms} / \mathrm{deg})$ & $0 / 0$ & $0 / 0.0315$ \\
\hline
\end{tabular}

Note that $G_{H}$ and $G_{A}$ values are given for resting/driven conditions.

membrane potential after repetitive firing and we note that previous results (Liu and Wang, 2001) have shown that our model is qualitatively similar to a previously proposed model of vestibular afferent activity based on a cumulative hyperpolarization (Smith and Goldberg, 1986). The synaptic current $I_{\text {synap }}$ is given by the following:

$$
\begin{aligned}
I_{\text {synap }} & =G_{H} \operatorname{HV}(t)-G_{A} X_{A}(t)+I_{\text {bias }}+\sigma \xi(t) \\
\dot{X}_{A} & =-\frac{X_{A}}{\tau_{A}}+\frac{\operatorname{HV}(t)}{\tau_{A}} .
\end{aligned}
$$

$\mathrm{HV}(t)$ is the horizontal head velocity, $I_{\text {bias }}$ is a bias current, $\sigma \xi(t)$ is Gaussian white noise with zero mean and SD $\sigma$, and $G_{H}$ is the intensity of the signal. Previous studies (Chacron et al., 2003) have shown that cumulative summation in the threshold variable after repetitive firing will lead to spike frequency adaptation and thus to increased gain at high stimulus frequencies. It is, however, known from experiments that irregular vestibular afferents have more high-pass transfer functions than regular ones (Goldberg, 2000); thus, we implemented a high-pass filter in the following way: the head velocity $\mathrm{HV}(t)$ is first low-pass filtered (cutoff frequency $1 / \tau_{A}$, where $\tau_{A}=20 \mathrm{~ms}$ ) to obtain $X_{A}$ and $G_{A} X_{A}$ is then subtracted from $G_{H} \mathrm{HV}(t) . G_{A}$ controls the amount of high-pass filtering: it is maximal when $G_{A}=G_{H}$ and null when $G_{A}=0$. We used low-pass filtered (eighth order Butterworth) white noise with cutoff frequency $f_{\mathrm{c}}=30 \mathrm{~Hz}$ and an SD of $20 \% \mathrm{~s}$ as in the data for $\mathrm{HV}(t)$. Numerical simulations were performed using an Euler-Maruyama integration algorithm with timestep $0.0025 \mathrm{~ms}$. Parameter values used for the simulations were taken to be within the physiological range (Tasaki, 1955; Smith and Goldberg, 1986) and are given in Table 1.

\section{Results}

\section{Spontaneous activity in regular and irregular afferents}

We recorded resting discharge from a total of 52 afferents from two macaque monkeys. Discharge regularity was estimated from a normalized coefficient of variation $\mathrm{CV}^{\star}$ (Goldberg et al., 1984) (see Materials and Methods). Thirty-one afferents were characterized as regularly firing $\left(\mathrm{CV}^{*}\right.$ mean $\left.=0.077 \pm 0.033\right)$, whereas the other 21 were characterized as irregularly firing $\left(\mathrm{CV}^{\star}\right.$ mean $=$ $0.344 \pm 0.116)$. Figure $1 a$ shows the ISI histogram obtained from the resting discharge (i.e., no stimulus) of a typical regular afferent. This afferent displayed low variability (Fig. $1 a$, inset) and this is reflected in the spike train power spectrum (Fig. $1 b$ ) by peaks at the resting firing rate $(\sim 95 \mathrm{~Hz})$ as well as integer multiples of that frequency (higher harmonics). Figure $1 c$ shows the ISI histogram obtained from the resting discharge for a typical irregular afferent. This afferent displayed considerable variability (Fig. 1c, inset) and its spike train power spectrum (Fig. 1d) showed considerably less structure consisting of a broader and shallower peak at its fundamental frequency $(\sim 90 \mathrm{~Hz})$ (Fig. $1 d)$. This irregular afferent displayed more power than the regular afferent over the behaviorally relevant frequency range $0-20 \mathrm{~Hz}$, as seen by comparing the power spectra. This difference is mostly caused by the differential variabilities of regular and irregular afferent resting 

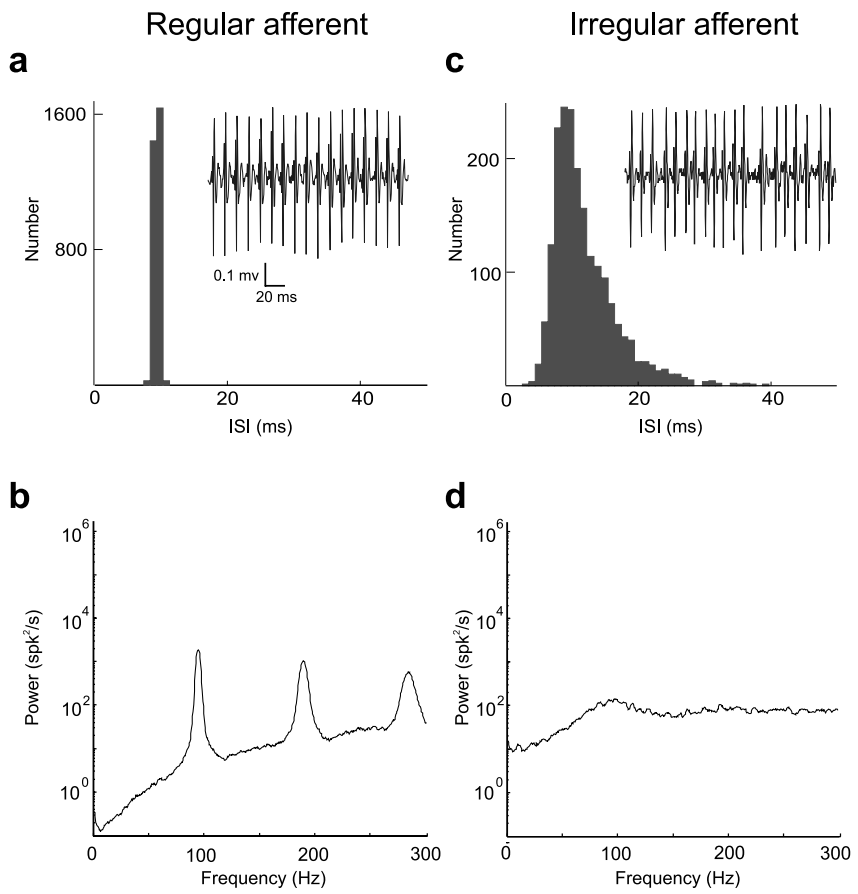

Figure 1. Spontaneous activity of typical regular and irregular afferents. $\boldsymbol{a}$, Interspike interval histogram from a typical regular afferent $\left(\mathrm{CV}^{*}=0.044\right)$. The inset shows an extracellular trace from that same afferent. $\boldsymbol{b}$, Spike train power spectrum for the regular afferent. The power spectrum shows low power at low frequencies and displays peaks at the resting discharge and its harmonics. $c$, Interspike interval histogram from a typical irregular afferent $\left(\mathrm{CV}^{*}=0.35\right)$. The inset shows an extracellular trace from that same afferent. $\boldsymbol{d}$, Spike train power spectrum for the irregular afferent. Note the higher power (i.e., higher variability) at low frequencies compared with the regular afferent.

discharges as quantified by CV. Indeed, it has been shown that the DC component of the power spectrum is directly related to $\mathrm{CV}$ (Cox and Lewis, 1966).

Previous theoretical studies have emphasized the role of the resting discharge on information transmission (Chacron et al., $2004,2005 a)$. In particular, the power spectrum of the resting discharge can be seen as a "noise spectrum" and determines the amount of noise at each frequency during stimulation. Lower noise over specific frequency ranges will give rise to higher signalto-noise ratios and, thus, better information transmission for these stimulus frequencies (Chacron et al., 2005a). The low power in the regular afferent's spike train over the behaviorally relevant frequency range $(0-20 \mathrm{~Hz})$ would thus predict better coding for behaviorally relevant head rotations. To verify this prediction, we next investigated the responses of regular and irregular afferents to sensory stimulation.

Information transmission by vestibular afferent spike trains Vestibular afferents were stimulated using broadband $(0-20 \mathrm{~Hz})$ noise with a Gaussian distribution of rotational head velocities (see Materials and Methods). The responses of 21 regular and 15 irregular vestibular afferents to these random stimuli were characterized using the stimulus reconstruction technique, which is based on an optimal linear decoding algorithm (Rieke et al., 1996) (see Materials and Methods). The quality of the reconstruction thus assesses the information carried by vestibular afferent spike trains. Figure $2 a$ shows the head velocity signal (gray line) as well as the reconstructed head velocity signal (dotted line) for an example regular afferent (same cell as Fig. 1a). As seen from a comparison between the original and reconstructed head veloc-

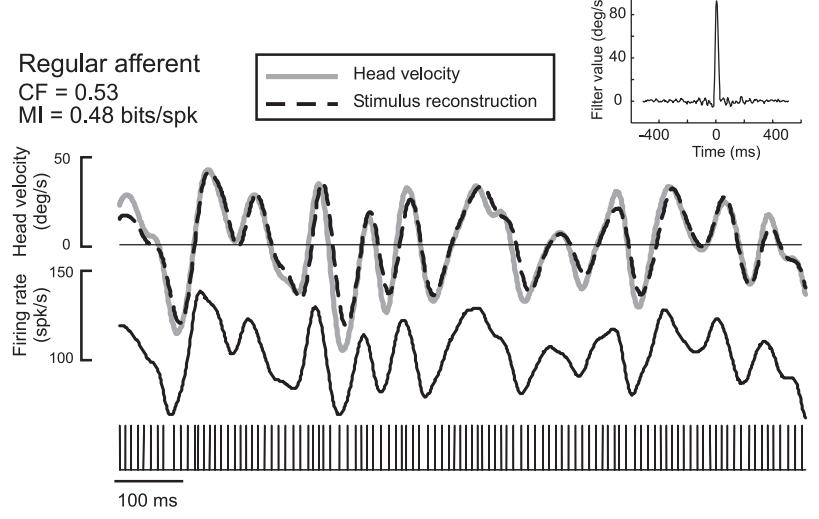

b

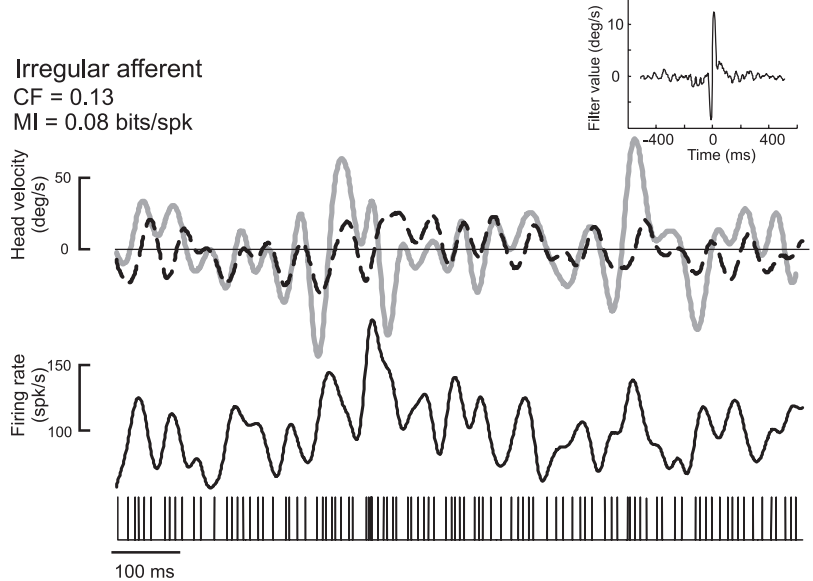

Figure 2. Stimulus reconstruction from example regular and irregular afferents in response to the $0-20 \mathrm{~Hz}$ broadband stimulus. $\boldsymbol{a}$, Gaussian random head velocity signal (gray) and reconstructed stimulus (black) for the regular afferent. The spike train and the time-dependent firing rate in response to the stimulus are also shown. The CF and Ml were both high. The inset shows the filter waveform that was used to estimate the head velocity from the spike train. $\boldsymbol{b}$, Gaussian random head velocity signal (gray) and reconstructed stimulus (black) for the regular afferent. The spike train and the time-dependent firing rate in response to the stimulus are also shown. The inset shows the filter waveform that was used to estimate the head velocity from the spike train. Both signals in $\boldsymbol{a}$ and $\boldsymbol{b}$ are $1 \mathrm{~s}$ samples from a $60 \mathrm{~s}$ recording.

ities, this regular afferent followed variations in the head velocity with great accuracy. Figure $2 b$ shows the reconstruction for an example irregular afferent (same cell as in Fig. $1 b$ ). This afferent was much less apt at reconstructing the detailed time course of the head velocity signal, as predicted from the power spectra of the resting discharges for the stimulus frequency range $(0-20 \mathrm{~Hz})$ (Fig. $1 b, d$ ). Whereas the reconstruction filter from the regular afferent was monophasic in shape and had a large amplitude (Fig. $2 a$, inset), the reconstruction filter from the irregular afferent was biphasic and had a lower amplitude (Fig. 2b, inset).

\section{This indicates important differences between both} afferent classes

We quantified the performance of regular and irregular afferents at reconstructing random head velocity stimuli by computing the coding fraction CF (i.e., the fraction of the stimulus that is correctly estimated) (see Materials and Methods). Regular afferents had significantly larger coding fractions than irregular afferents $\left(\mathrm{CF}_{\text {reg }}=0.39 \pm 0.13 ; \mathrm{CF}_{\text {irreg }}=0.24 \pm 0.08 ; p=0.0002, t\right.$ test; $\mathrm{df}=36$ ). We also computed the mutual information rate MI (see Materials and Methods). Regular afferents transmitted on average two times more information per spike than irregular afferents 
a



b

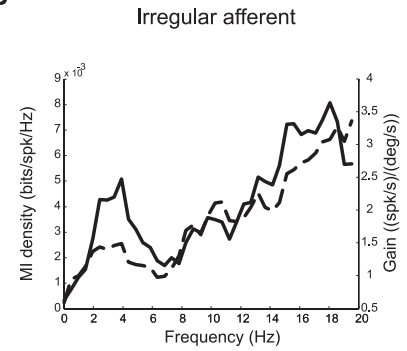

a

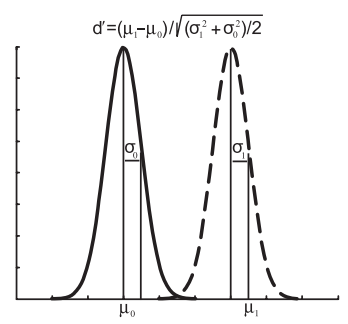

C

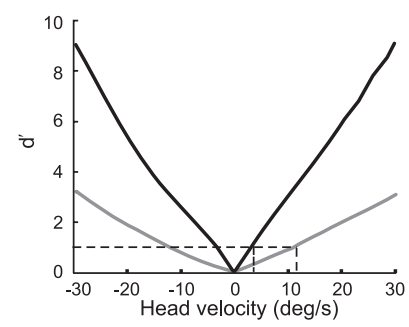

b

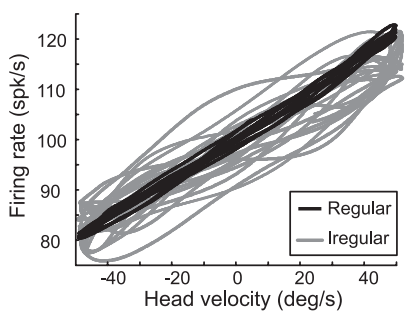

d

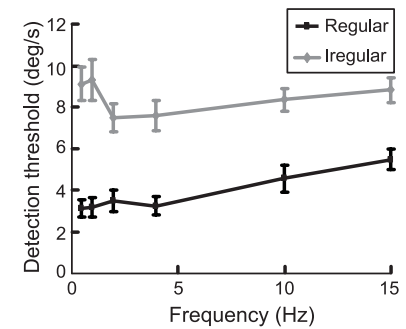

inform 3. Comparison between mutual information measures and gain measures. $\boldsymbol{a}$, Mutua The gain curve increased as a function of frequency, whereas the mutual information density was constant. $\boldsymbol{b}$, Mutual information density (solid line) and gain (dotted line) as a function of frequency for the irregular afferent. The gain and mutual information density curves had similar shapes and increased with frequency.
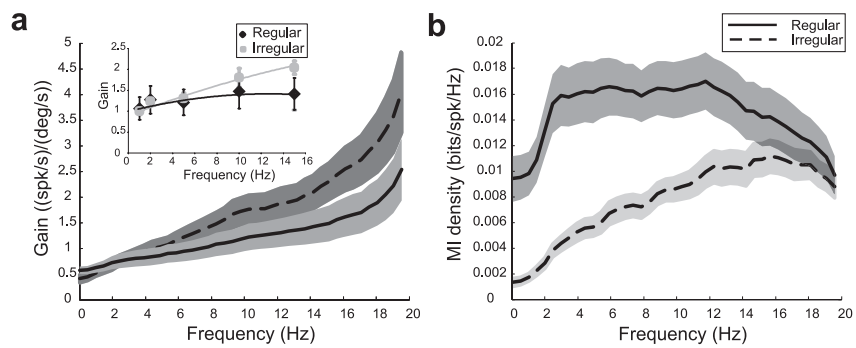

Figure 4. Gain and mutual information density for populations of vestibular afferents. $\boldsymbol{a}$, Population-averaged gains as a function of frequency for regular (solid line) and irregular (dotted line) afferents obtained during random head rotations. The gray bands show 1 SEM. The inset shows population averaged gains as a function of frequency for sinusoidal head rotations with error bars showing 1 SEM. Irregular afferents had higher gains for higher frequencies. $\boldsymbol{b}$, Population-averaged mutual information density curves for regular (solid line) and irregular (dotted line) afferents. These were relatively constant for regular afferents and increased with frequency for irregular afferents.

$\left(\mathrm{MI}_{\text {reg }}=0.36 \pm 0.25\right.$ bits/spike; $\mathrm{MI}_{\text {irreg }}=0.18 \pm 0.08$ bits $/$ spike; $p=0.0038, t$ test; $\mathrm{df}=36$ ).

\section{Comparison with more traditional measures}

We compared information theoretic quantities to measures of gain that have been traditionally used to characterize vestibular afferent responses. Figure $3 a$ shows the mutual information rate density (i.e., the mutual information per spike per frequency; solid line) as well as the gain (dotted line) for an example regular afferent (same cell as in Fig. 1a). Note that our information rates were normalized to account for firing rate differences among different units (see Materials and Methods). This regular afferent displayed a gain that increased as a function of frequency. However, the information rate density curve was constant over the frequency range of $0-20 \mathrm{~Hz}$. Figure $3 b$ shows the same curves for an example irregular afferent (same cell as Fig. 1b) and, in contrast to Figure $3 a$, the information rate density curve had a similar shape to that of the gain curve.

These results were consistently seen across the population studied. Population-averaged gain curves for regular (solid) and irregular (dotted) afferents are shown in Figure $4 a$ for random head rotations. For both classes, the gain increased with frequency and the average gains between $0.5-5 \mathrm{~Hz}\left(G_{\text {low }}\right)$ and $15-20$ $\mathrm{Hz}\left(G_{\text {high }}\right)$ were significantly different for both regular $(p<$ $10^{-3}$, pairwise $t$ test; $\left.n=21\right)$ and irregular $\left(p<10^{-3}\right.$, pairwise $t$ test; $n=15$ ) afferents. These results were not an artifact of using random head rotations because the gain values obtained from
Figure 5. Detection thresholds for regular and irregular afferents. $\boldsymbol{a}$, Schematic representation of the firing-rate distribution during resting discharge (solid line) and during stimulation (dashed line). $\boldsymbol{b}$, Plot of firing rate as a function of head velocity for an example regular (black) and irregular (gray) afferent during sinusoidal rotation of $0.5 \mathrm{~Hz}$. Note the strong correlation between both quantities for the regular afferent. $c$, Values of $d^{\prime}$ obtained for the example regular (black) and irregular (gray) afferents during a $0.5 \mathrm{~Hz}$ sinusoidal rotation. $A d^{\prime}$ of 1 was used for computing the detection threshold velocities for each afferent (dotted lines). $\boldsymbol{d}$, Population-averaged detection threshold values for regular (black; $n=10$ ) and irregular (gray; $n=10$ ) units at different frequencies of sinusoidal head rotation. Error bars indicate SEM.

random head rotations showed trends similar to those obtained with more traditional sinusoidal head rotations (Fig. $4 a$, inset).

In contrast to gain measures, mutual information density measures obtained from regular and irregular afferents showed qualitative differences (Fig. 4b). Irregular afferents transmit more information about high frequencies whereas regular afferents are tuned both to low and high frequencies. We computed the average information rate densities between $0.5-5 \mathrm{~Hz}\left(M_{\text {low }}\right)$ and $15-20 \mathrm{~Hz}\left(M_{\text {high }}\right)$ to assess coding within the low and high ends of the behaviorally relevant frequency range of $0-20 \mathrm{~Hz}$. Although these were significantly different for irregular afferents $(p<$ $10^{-3}$, pairwise $t$ test; $\left.n=15\right)$, no difference was seen for regular afferents ( $p=0.77$, pairwise $t$ test; $n=21$ ). The difference between the gain and mutual information density curves for regular afferents most likely originates from the low variability displayed by their resting discharge (Fig. 1a,b), as suggested by previous theoretical studies (Chacron et al., 2004; Lindner et al., 2005).

\section{Detection thresholds for regular and irregular afferents}

The higher information rates obtained under broadband (0-20 $\mathrm{Hz}$ ) stimulation for regular afferents suggest that they may be able to detect smaller amplitude head velocities than irregular afferents. We thus used the $\mathrm{d}^{\prime}$ measure from signal detection theory (Green and Swets, 1966) to discriminate between the firing rate distribution obtained under resting discharge and that obtained under sinusoidal head rotations at different frequencies (Fig. 5a). We assessed the relationship between head velocity and firing rate for each afferent type by plotting the time-dependent firing rate as a function of the head velocity signal (Fig. $5 b$ ). For a given head velocity, there was significantly less variability in the firing rate of regular afferents (Fig. $5 b$, dark) as compared with irregular afferents (Fig. 5b, gray). As a consequence, the d' mea- 
sure increased as a function of head velocity with a greater rate (Fig. 5c, compare black and gray) for regular afferents. We computed the discrimination threshold of each afferent as the minimum absolute head velocity for which $\mathrm{d}^{\prime}=1$ (Fig. $5 c$ ). For our two examples, the regular afferent had a detection threshold of $3.7 \%$ and the irregular afferent had a much higher detection threshold of $12.4 \%$ s. Similar results were seen across the population studied because regular afferents consistently had lower detection thresholds $(4.0 \pm 1.0 \%)$ than irregular afferents $(8.4 \pm$ $0.7 \%$ ) over behaviorally relevant frequencies ranging from 0.5 to $15 \mathrm{~Hz}$ (Fig. $5 d)(p<0.001, t$ tests; $n=20)$.

\section{Modeling regular and irregular afferents}

To gain insight as to the possible differences between regular and irregular afferents that could explain their different coding properties, we built a simple phenomenological model of vestibular afferent activity. The model consists of two variables: the membrane potential and threshold. Action potentials are produced when the voltage reaches the threshold (see Materials and Methods). After each action potential, the voltage is reset while the threshold is incremented by a constant amount, which means that rapid firing can lead to cumulative summation in the threshold. We note that this same model has been used to successfully reproduce data from weakly electric fish electroreceptor afferents (Chacron et al., 2001) and that the model structure is qualitatively similar to that of the model proposed previously by Smith and Goldberg (1986) to account for the resting discharges of vestibular afferents.

We found parameter sets for which the model accurately reproduced experimental results for each afferent class under resting conditions (i.e., no stimulus present) (see Materials and Methods). Results from the resting discharge of model regular and irregular afferents are shown in Figure 6. For the regular afferent model, the ISI histogram (Fig. 6a) and spike train power spectrum (Fig. 6b) closely resemble those obtained experimentally (compare Fig. 1a, b, respectively). The model displayed quasi-periodic firing (Fig. $6 a$, inset).

To model irregular vestibular afferents, we increased spike train variability by increasing the noise intensity in the model relative to the value for regular afferents. The ISI histogram (Fig. $6 c$ ) and spike train power spectrum (Fig. $6 d$ ) obtained with the irregular afferent model closely resembled those obtained experimentally (compare Fig. 1c, d, respectively). The model displayed significantly less periodic firing (Fig. $6 c$, inset). Overall, $\mathrm{CV}^{\star}$ increased as a function of increasing noise intensity (Fig. 6e). Thus, these modeling results suggest that internal noise is a major factor contributing to the differences between the resting discharges of regular and irregular afferents, which is consistent with previous results (Goldberg and Fernández, 1971a; Smith and Goldberg, 1986).

Results obtained for the model regular and irregular afferents under driven conditions (i.e., in the presence of a stimulus) are shown in Figure 7, $a$ and $b$, respectively. The gain and mutual information density curves closely resembled those obtained experimentally (compare Fig. 3). To gain insight as to the role of internal noise on information transmission about sensory stimuli, we systematically varied the internal noise intensity $\sigma$. We varied this parameter in both the regular and irregular afferent models to eliminate the potential confounding effects of other differences such as the high-pass filter used for the irregular afferent model (see Materials and Methods). In particular, we verified the prediction made previously that increasing noise intensity should decrease the signal-to-noise ratio and, thus, infor-

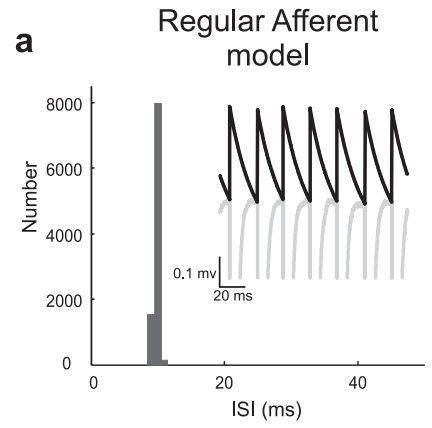

C

Irregular Afferent

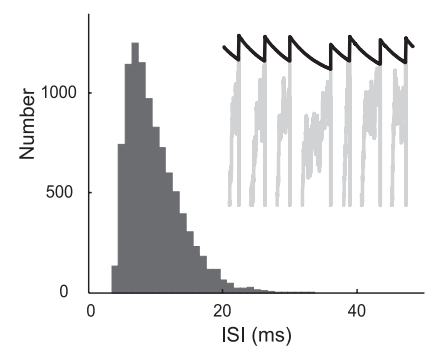

b

d
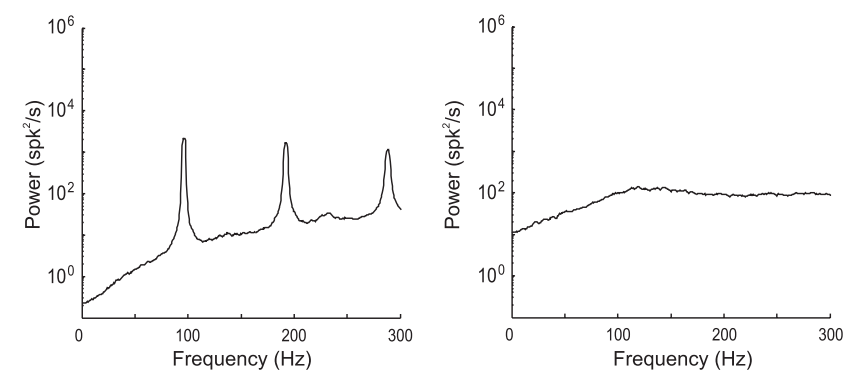

e

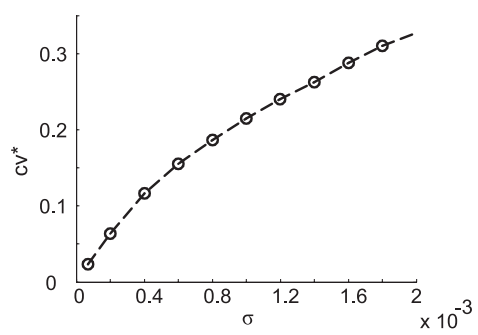

Figure 6. Models of spontaneous activity in regular and irregular afferents. $\boldsymbol{a}$, Interspike interval histogram obtained with the regular afferent model. The inset shows the voltage (gray) and threshold (black) variables of the model as a function of time. $\boldsymbol{b}$, Spike train power spectrum for the regular afferent model. $c$, Interspike interval histogram obtained with the irregular afferent model. The inset shows the voltage (gray) and threshold (black) variables of the model as a function of time. $\boldsymbol{d}$, Spike train power spectrum for the irregular afferent model. $\boldsymbol{e}, \mathrm{CV}^{*}$ as a function of noise intensity $\sigma$ showing the transition between regularity and irregularity. Comparison with Figure 1 shows that the model can reproduce the salient features of the experimental data.

mation transmission as results showed that increasing the noise intensity $\sigma$ decreased information transmission for low frequencies $\left(M_{\text {low }}\right)$ and high frequencies $\left(M_{\text {high }}\right)$ in both models (Fig. $7 c, d)$.

In contrast, measures of gain at low frequencies $\left(G_{\text {low }}\right)$ and high frequencies $\left(G_{\text {high }}\right)$ were insensitive to variations in $\sigma$ for both models (Figs. $7 c, d$ ). This is expected because they are obtained by averaging out neural variability and should thus be relatively insensitive to the overall amount of noise in the model (Rieke et al., 1996; Chacron et al., 2005a). Mutual information is a direct function of the signal-to-noise ratio (Rieke et al., 1996) and is thus sensitive to the overall amount of noise in the model, as confirmed by our results. Our modeling results thus provide an explanation for the difference between gain and information theoretic measures seen in regular afferents as well as the lack of difference for irregular afferents.

We also computed detection thresholds for the regular and irregular afferent models. Increasing the noise intensity $\sigma$ should give rise to increased detection thresholds because of increased 

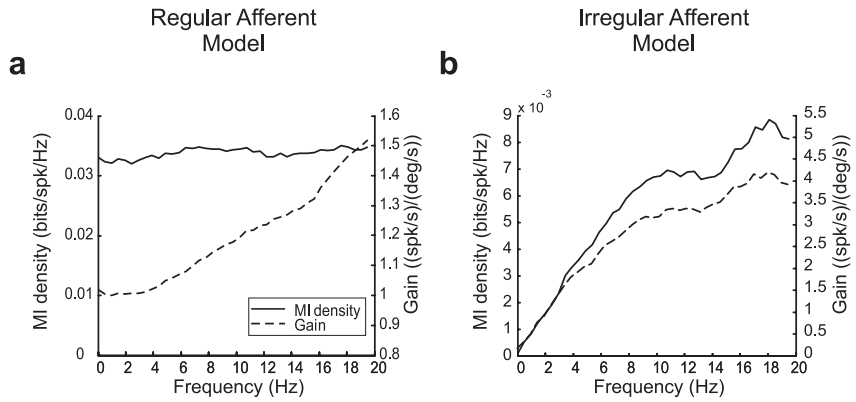

C

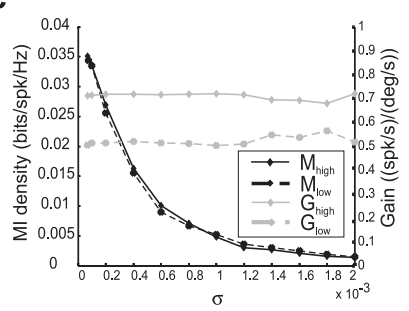

d

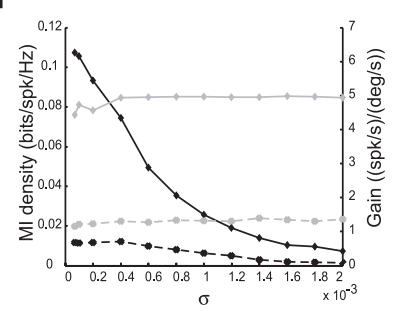

e

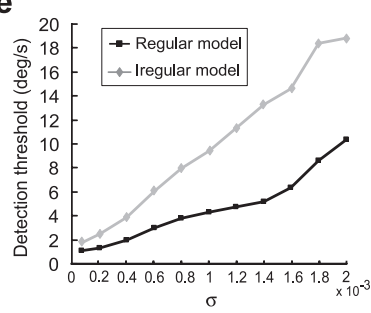

Figure 7. The role of intrinsic noise on information processing and signal detection. $\boldsymbol{a}, \mathrm{Mu}-$ tual information density (solid line) and gain curve (dotted line) obtained with the regular afferent model under driven conditions. The mutual information curve was constant over frequency, whereas the gain curve increased with frequency. $\boldsymbol{b}$, Mutual information density (solid line) and gain curve (dotted line) obtained with the irregular afferent model under driven conditions. Both the mutual information density and the gain curve increased with frequency.c, Low-frequency (dotted black line) and high-frequency (solid black line) average mutual information densities as a function of noise intensity $\sigma$ for the regular afferent model. Both quantities decreased as $\sigma$ increased. In contrast, the low-frequency (dotted gray line) and highfrequency (solid gray line) averaged gains were unaffected by variations in $\sigma . \boldsymbol{d}$, Low-frequency (dotted black line) and high-frequency (solid black line) average mutual information densities as a function of noise intensity $\sigma$ for the irregular afferent model. Both quantities decreased as $\sigma$ increased. In contrast, the low-frequency (dotted gray line) and high-frequency (solid gray line) averaged gains were unaffected by variations in $\sigma . \boldsymbol{e}$, Detection threshold velocities of regular afferent model (black line) and irregular afferent model (gray line) as a function of noise intensity $\sigma$. The detection threshold increased with increasing noise intensity for both models.

variability and this is indeed the case for both models (Fig. 7e). Increasing $\sigma$ from values used to model regular afferents $(0.00007)$ to values used to model irregular afferents (0.0015) gave rise to increases in detection threshold from values typically seen for regular afferents $(2 \%)$ to values typically seen for irregular afferents $(15 \%)$. As such, our modeling results can also explain the differences between regular and irregular afferents in terms of detection threshold.

\section{Neural variability and the nature of the neural code}

Together, the above results show that regular afferents transmit more information and have lower detection thresholds than irregular afferents. Although these results show that neural variability influences sensory coding in vestibular afferents, they do not explicitly establish the nature of the neural codes used by these afferents. Neural codes can be differentiated into two categories: rate neural codes in which all of the information is carried by the time-dependent firing rate and temporal codes in which at least some of the information is carried by the actual timings of action potentials (Adrian and Zotterman, 1926; Theunissen and Miller, 1995; Stein et al., 2005). Because temporal codes rely on the precise timing of action potentials, the information transmitted should be significantly altered when the timing becomes less precise (Theunissen and Miller, 1995). For rate codes, however, the information transmitted should be independent of spike timing precision because action potential timing itself carries no information.

To test for the presence of temporal codes in the peripheral vestibular system, we added random spike-timing jitter to the spike trains obtained from both regular and irregular afferents (Fig. 8). We note that this approach has already been used previously to establish the presence of temporal codes in other systems (Jones et al., 2004). Because the jitter time scale used (2 ms) was much smaller than the noise stimulus time scale (50 ms), addition of jitter was not expected to significantly alter information encoded by the time-dependent firing rate. This was confirmed by plotting the gain curve, which was not significantly affected by addition of spike-timing jitter for the regular afferent (Fig. 8a). However, the mutual information density curve of the regular afferent was reduced by a significant amount at low frequencies $(<2 \mathrm{~Hz})$ whose inverse $(>500 \mathrm{~ms})$ was much greater than either the jitter or the stimulus time scales (Fig. $8 b$ ). This indicates that a significant fraction of the information transmitted by this regular afferent was carried in the precise timing of action potentials. The quality of the stimulus reconstruction, as quantified by the coding fraction, was reduced by the addition of spike-timing jitter by $34 \%$ (Fig. $8 e$, top), whereas the time-dependent firing rate was unaffected (Fig. 8e, bottom), which further supports the presence of a temporal code for this regular afferent.

The situation was, however, different for the example irregular afferent. Although addition of spike-timing jitter had no effect on the gain curve (Fig. $8 c$ ), the effect on the mutual information density curve was small. Furthermore, the effect was negligible for low frequencies $(<2 \mathrm{~Hz})$ whose inverse $(>500 \mathrm{~ms})$ was much greater than either the jitter or stimulus time scales (Fig. $8 d$ ). Addition of spike-timing jitter also had no significant effect on the quality of stimulus reconstruction (Fig. $8 f$, top, compare red and blue curves) and the coding fraction was reduced by a mere $1.6 \%$ for this particular afferent. Finally, addition of spike-timing jitter had negligible effects on time dependent firing rate for the irregular afferent (Fig. $8 f$, bottom, compare red and blue curves). These results strongly suggest that action potential timing carried little, if any, information for this irregular afferent and argue for the presence of a rate code.

Similar results were consistently observed across our populations of regular and irregular afferents. Addition of jitter had negligible effects on the gain at low frequencies as $G_{\text {low }}$ varied only by $1.53 \pm 5.65 \%$ for regular afferents and by $0.88 \pm 4.94 \%$ for irregular afferents. The low-frequency information $\mathrm{MI}_{\text {low }}$ was, however, reduced on average by $21.55 \pm 18.41 \%$ for regular afferents and only by $3.86 \pm 5.30 \%$ for irregular afferents. Moreover, the quality of stimulus reconstruction as quantified by the coding fraction was reduced on average by $28.12 \pm 16.37 \%$ for regular afferents and by $9.07 \pm 3.06 \%$ for irregular afferents. Comparable effects were obtained using our model (supplemental Fig. 1, available at www.jneurosci.org as supplemental material). Thus, our experimental and modeling results strongly suggest that neural variability has a strong influence on the coding schemes used by the peripheral vestibular system: regular afferents use temporal codes whereas irregular afferents use rate codes. 


\section{Discussion}

Summary of results

We studied vestibular afferent responses to sinusoidal and random head rotations using gain, information, and signal detection measures. Regular afferents had higher information rates and lower detection thresholds over a wide range of behaviorally relevant $(0-20 \mathrm{~Hz})$ frequencies as compared with irregular afferents. In contrast, measures of gain predicted better responses for irregular afferents over the same frequency range. A simple leaky integrate-and-fire model with a dynamic threshold could reproduce these results and suggested that neural variability in vestibular afferents is a function of intrinsic noise. By adding spike-timing jitter, we showed that neural variability has a significant effect on the nature of the neural code. We found that temporal codes are present in the peripheral vestibular system as information is carried in the spiking times of regular afferents only.

\section{Neural variability and}

information transmission

We quantified the frequency responses of vestibular afferents to novel random as well as traditional sinusoidal head rotations within the behaviorally relevant frequency range $0-20 \mathrm{~Hz}$ (Grossman et al., 1988; Armand and Minor, 2001; Huterer and Cullen, 2002). Our results with sinusoidal stimuli confirmed those of previous studies showing that, although irregular and regular afferents have comparable gains at frequencies of $0.5 \mathrm{~Hz}$, irregular afferents consistently displayed higher gains with increasing frequency (Goldberg and Fernandez, 1971b; Baird et al., 1988; Hullar and Minor, 1999; Goldberg, 2000; Haque et al., 2004; Hullar et al., 2005; Ramachandran and Lisberger, 2006; Sadeghi et al., 2006). Moreover, random stimuli gave rise to qualitatively similar gains, which is consistent with the traditional linear system's approach applied to the vestibular system. Given that irregular afferents have gains that are greater or equal to those of regular afferents over the physiological frequency range of natural head movements, one might question the reasons for having regular vestibular afferents at all.

In the present study, we applied, for the first time, information theoretic measures to characterize the responses of vestibular afferents to random head rotations over the behaviorally relevant frequency range of $0-20 \mathrm{~Hz}$. Contrary to predictions from gain, regular afferents consistently transmitted more information than irregular afferents for low-frequency head rotations $(<5 \mathrm{~Hz})$. This result may have important consequences for the functional roles of each

e

f a

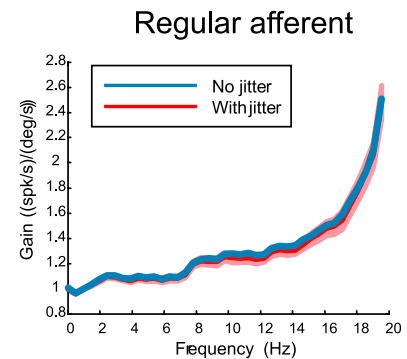

b

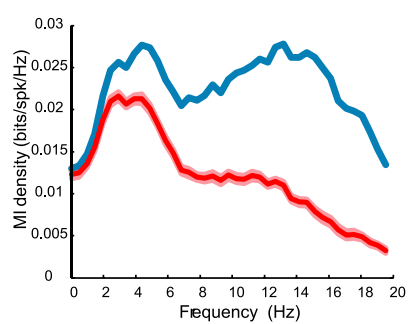

C

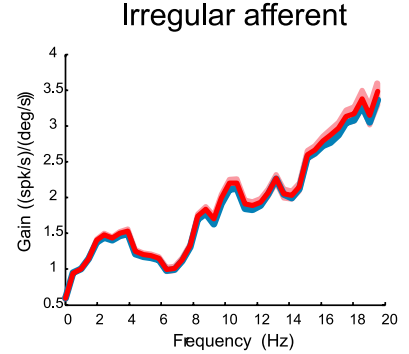

d

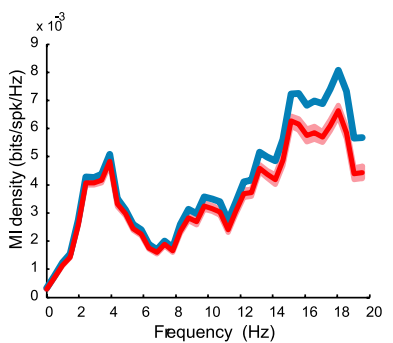

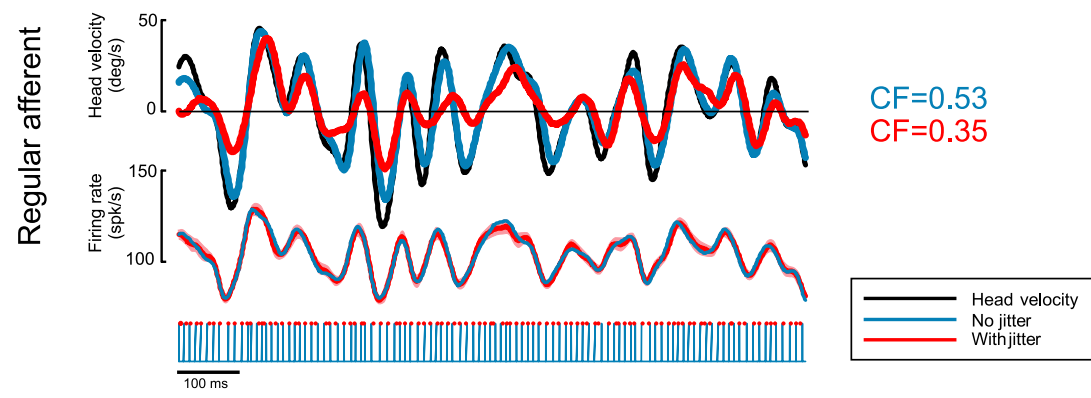

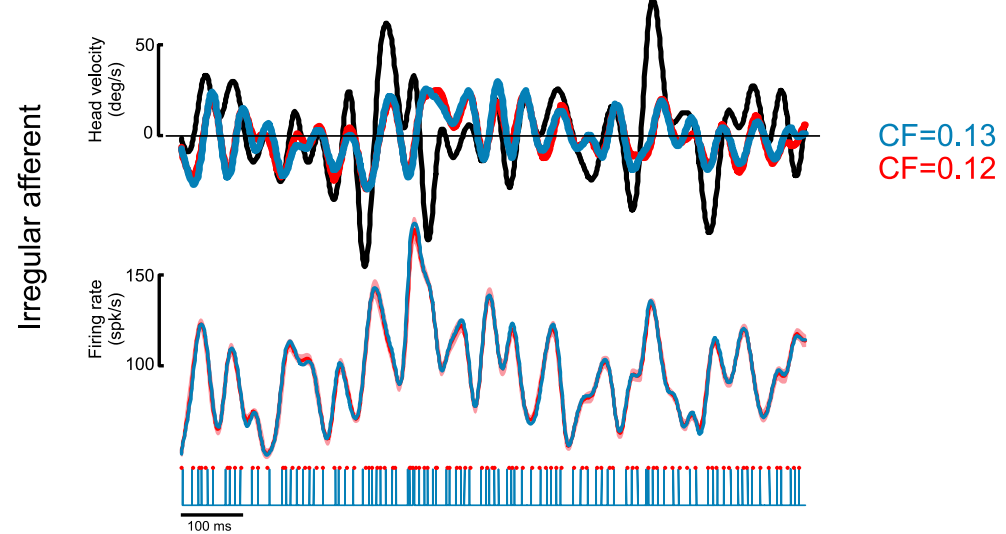

Figure 8. Effects of spike-timing jitter on gain and mutual information density of regular and irregular afferents. $\boldsymbol{a}$, Gain curve with (red) and without (blue) addition of $2 \mathrm{~ms}$ jitter for the regular afferent. Addition of jitter had no significant effect on gain. $\boldsymbol{b}$, Mutual information density curve obtained with (red) and without (blue) addition of $2 \mathrm{~ms}$ jitter for the regular afferent. Addition of jitter decreased the mutual information significantly. $c$, Gain curve with (red) and without (blue) addition of $2 \mathrm{~ms}$ jitter for the irregular afferent. Addition of jitter had no significant effect on gain. $\boldsymbol{d}$, Mutual information density curve obtained with (red) and without (blue) addition of $2 \mathrm{~ms}$ jitter for the irregular afferent. Addition of jitter had a much smaller effect on the information density compared with the regular afferent. $\boldsymbol{e}$, Top, Original head velocity (black) and reconstructed head velocities with (red) and without (blue) 2 ms spike-timing jitter. Middle, Time-dependent firing rates obtained with (red) and without (blue) addition of 2 ms spike-timing jitter for the regular afferent. Bottom, Jittered (red dots) and unjittered (blue lines) spike times. f, Top, Original head velocity (black) and reconstructed head velocities with (red) and without (blue) 2 ms spike-timing jitter. Middle, Timedependent firing rates obtained with (red) and without (blue) addition of $2 \mathrm{~ms}$ spike-timing jitter for the irregular afferent. Bottom, Jittered (red dots) and unjittered (blue lines) spike times. The light red bands represent 1 SEM obtained from averaging over repeated realizations of the jitter (see Materials and Methods).

afferent class. For example, the compensatory eye movements produced by the vestibulo-ocular reflex (VOR) (Huterer and Cullen, 2002) requires that vestibular afferents encode the time course of head rotations. Regular afferents make the primary 
contribution to the VOR for frequencies $<4 \mathrm{~Hz}$ (Minor and Goldberg, 1991; Chen-Huang et al., 1997). This is consistent with our results showing that regular afferents transmitted more information about low-frequency head rotations than irregular afferents.

In contrast, the mutual information density and gain curves for irregular afferents were both minimal at low frequencies and increased as a function of frequency. This suggests that irregular afferents play a greater role in vestibular processing of highfrequency head rotations, as suggested by previous studies (Goldberg and Fernandez, 1971a,b; Hullar et al., 2005; Ramachandran and Lisberger, 2006; Sadeghi et al., 2006). Another proposed function for irregular afferents is to mediate behaviorally appropriate changes in the VOR such as those necessitated by changes in viewing distance (Chen-Huang et al., 1997). Additional studies are needed to understand the mechanisms by which vestibular nuclei (VN) neurons integrate inputs from both afferent classes to achieve this modulation.

\section{Neural variability and signal detection}

The higher information rates of regular afferents lead us to hypothesize that they would be better at detecting small perturbations. Consistent with our predictions, we found that regular afferents had detection thresholds that were approximately half of those of irregular afferents. Previous results show behavioral detection thresholds of $\geq 1 \%$ for angular movements at low frequencies of 0.025-0.45 Hz (Mergner et al., 1993; Becker et al., 2000). Our detection thresholds for single afferents are larger, indicating that higher-order neurons must integrate inputs from vestibular afferent populations as well as other sensory modalities (Benson et al., 1989; Mergner et al., 1993; Fitzpatrick and McCloskey, 1994; Bacsi and Colebatch, 2005; Jurgens and Becker, 2006).

\section{Morphological differences and cellular mechanisms underlying discharge variability}

Together, our results suggest that the central vestibular system receives input from two different information channels. This is further supported by previous studies showing morphological correlates for vestibular afferent discharge variability. Afferents that terminate as calyx endings onto type I hair cells in the central zone of the crista (the sensory neuroepithelium of the canals) have irregular discharges. Regular afferents, in contrast, contact type II hair cells with bouton endings in the peripheral zone (Baird et al., 1988). Dimorphic afferents make both calyx endings onto type I hair cells and bouton endings onto type II hair cells. More recent work suggests that cellular mechanisms such as potassium channel densities contribute to the differences between regular and irregular afferents (Eatock et al., 2002; Eatock and Hurley, 2003; Limón et al., 2005; Hurley et al., 2006).

\section{Role of intrinsic noise, variability, and system nonlinearities in sensory coding}

Although neurons are by definition nonlinear encoding devices because of their spiking mechanism, linear encoding models are often adequate for weak (i.e., low intensity) sensory stimuli (Roddey et al., 2000; Chacron et al., 2004). These models have been applied to vestibular afferents (Goldberg and Fernandez, 1971b; Baird et al., 1988; Hullar and Minor, 1999; Haque et al., 2004; Hullar et al., 2005). Such models will underestimate the amount of information transmitted by regular afferents, because $30 \%$ of the information is carried in action potential timing. Thus, regular afferents can be modeled more efficiently by nonlinear processes such as the one proposed here. However, previous studies have shown that noise linearizes nonlinear systems (Chacron et al., 2005c; Lindner et al., 2005), which might explain why linear encoding models are generally adequate to describe responses of irregular afferents to sensory input.

Previous studies in other systems have shown that information encoded in a nonlinear manner can sometimes be decoded through linear algorithms (Rieke et al., 1996). Our results show that this is the case for regular afferents because they were shown to encode information through a temporal code yet displayed high coding fractions and mutual information rates. We note that linear decoding algorithms perform poorly when significant nonlinearities are present (Roddey et al., 2000). The low coding fractions and information rates observed for irregular afferents are most likely not attributable to static nonlinearities because our stimuli were within the linear encoding range (Hullar and Minor, 1999; Hullar et al., 2005; Sadeghi et al., 2006) where no phase-locking occurs (Ramachandran and Lisberger, 2006). Rather, our modeling results suggest that they are caused by high levels of intrinsic noise, which will also limit the performance of linear decoders (Roddey et al., 2000).

Because regular afferents display low levels of intrinsic noise, it is possible that nonlinear decoding algorithms will outperform the optimal linear algorithms used here. In fact, VN neurons receiving direct synaptic input from vestibular afferents possess subthreshold voltage-gated ion currents such as persistent sodium (Johnston et al., 1994; Beraneck et al., 2003; Straka et al., 2005) that lead to amplification of coincident synaptic input (Stuart and Sakmann, 1995; Crill, 1996; Berman and Maler, 1998; Andreasen and Lambert, 1999). It is thus possible that VN neural responses depend critically on the precise timing of synaptic input.

\section{General strategies for sensory coding}

A simple leaky integrate-and-fire model with a dynamic threshold could explain the differences between regular and irregular vestibular afferents. Our modeling results, like those of Smith and Goldberg (1986), suggest that intrinsic noise is a major contributor to the differences in the resting discharge variabilities of regular and irregular afferents. The increase in threshold after each action potential, which mimics activation of an afterhyperpolarizing potassium, was larger for the regular afferent model than for the irregular afferent model, consistent with experimental findings (Schessel, 1982; Schessel et al., 1991). Furthermore, our model has been able to characterize the responses of electroreceptor afferents in weakly electric fish (Chacron et al., 2000, 2001). This is not unexpected as both systems have presumably evolved from the lateral line (Romer and Parsons, 1977). However, although electroreceptor afferents have similar information tuning to regular vestibular afferents (Chacron et al., 2005a), temporal codes are not present in electroreceptor afferents (Kreiman et al., 2000; Chacron, 2006).

Temporal codes have also been demonstrated in the visual (Richmond et al., 1987; Meister, 1996), auditory (Machens et al., 2001), tactile (Johansson and Birznieks, 2004; Jones et al., 2004), and olfactory (Laurent and Davidowitz, 1994) systems. However, all but one of these studies focused on higher processing levels in the brain. One study (Johansson and Birznieks, 2004) has shown that information was contained in the timings of spike trains in the human tactile afferents, which contradicts the assumption that peripheral sensory neurons employ a rate code. Our results are the first to show that both spike timing and rate codes can coexist at the sensory periphery and may provide an explanation 
for the presence of a heterogeneous peripheral sensory neuron population.

\section{References}

Adrian E, Zotterman Y (1926) The impulses produced by sensory nerve endings. Part 3. Impulses set up by touch and pressure. J Physiol (Lond) 61:465-483.

Andreasen M, Lambert JD (1999) Somatic amplification of distally generated subthreshold EPSPs in rat hippocampal pyramidal neurones. J Physiol (Lond) 519:85-100.

Armand M, Minor LB (2001) Relationship between time- and frequencydomain analyses of angular head movements in the squirrel monkey. J Comput Neurosci 11:217-239.

Bacsi AM, Colebatch JG (2005) Evidence for reflex and perceptual vestibular contributions to postural control. Exp Brain Res 160:22-28.

Baird RA, Desmadryl G, Fernandez C, Goldberg JM (1988) The vestibular nerve of the chinchilla. II. Relation between afferent response properties and peripheral innervation patterns in the semicircular canals. J Neurophysiol 60:182-203.

Becker W, Jurgens R, Boss T (2000) Vestibular perception of self-rotation in different postures: a comparison between sitting and standing subjects. Exp Brain Res 131:468-476.

Benson AJ, Hutt EC, Brown SF (1989) Thresholds for the perception of whole body angular movement about a vertical axis. Aviat Space Environ Med 60:205-213.

Beraneck M, Hachemaoui M, Idoux E, Ris L, Uno A, Godaux E, Vidal PP, Moore LE, Vibert N (2003) Long-term plasticity of ipsilesional medial vestibular nucleus neurons after unilateral labyrinthectomy. J Neurophysiol 90:184-203.

Berman NJ, Maler L (1998) Distal versus proximal inhibitory shaping of feedback excitation in the electrosensory lateral line lobe: implications for sensory filtering. J Neurophysiol 80:3214-3232.

Borst A, Haag J (2001) Effects of mean firing on neural information rate. J Comp Neurosci 10:213-221.

Carr CE (1993) Processing of temporal information in the brain. Annu Rev Neurosci 16:223-243.

Carr CE, Konishi M (1990) A circuit for detection of interaural time differences in the brain stem of the barn owl. J Neurosci 10:3227-3246.

Chacron MJ (2006) Nonlinear information processing in a model sensory system. J Neurophysiol 95:2933-2946.

Chacron MJ, Longtin A, St-Hilaire M, Maler L (2000) Suprathreshold stochastic firing dynamics with memory in P-type electroreceptors. Phys Rev Lett 85:1576-1579.

Chacron MJ, Longtin A, Maler L (2001) Negative interspike interval correlations increase the neuronal capacity for encoding time-varying stimuli. J Neurosci 21:5328-5343.

Chacron MJ, Pakdaman K, Longtin A (2003) Interspike interval correlations, memory, adaptation, and refractoriness in a leaky integrate-andfire model with threshold fatigue. Neural Comput 15:253-278.

Chacron MJ, Lindner B, Longtin A (2004) Noise shaping by interval correlations increases information transfer. Phys Rev Lett 92:080601.

Chacron MJ, Maler L, Bastian J (2005a) Electroreceptor neuron dynamics shape information transmission. Nat Neurosci 8:673-678.

Chacron MJ, Maler L, Bastian J (2005b) Feedback and feedforward control of frequency tuning to naturalistic stimuli. J Neurosci 25:5521-5532.

Chacron MJ, Longtin A, Maler L (2005c) Delayed excitatory and inhibitory feedback shape neural information transmission. Phys Rev E Stat Nonlin Soft Matter Phys 72:051917.

Chen-Huang C, McCrea RA, Goldberg JM (1997) Contributions of regularly and irregularly discharging vestibular-nerve inputs to the discharge of central vestibular neurons in the alert squirrel monkey. Exp Brain Res 114:405-422.

Connor CE, Hsiao SS, Phillips JR, Johnson KO (1990) Tactile roughness: neural codes that account for psychophysical magnitude estimates. J Neurosci 12:3823-3826.

Cox DR, Lewis PA (1966) The statistical analysis of series of events. London: Methuen.

Crill WE (1996) Persistent sodium current in mammalian central neurons. Annu Rev Physiol 58:349-362.

Cullen KE, Minor LB (2002) Semicircular canal afferents similarly encode active and passive head-on body rotations: implications for the role of vestibular efference. J Neurosci 22:RC226.
Dayan P, Abbott LF (2001) Theoretical neuroscience: computational and mathematical modeling of neural systems. Cambridge, MA: MIT.

Eatock RA, Hurley KM (2003) Functional development of hair cells. Curr Top Dev Biol 57:389-448.

Eatock RA, Hurley KM, Vollrath MA (2002) Mechanoelectrical and voltage-gated ion channels in mammalian vestibular hair cells. Audiol Neurootol 7:31-35.

Fernandez C, Baird RA, Goldberg JM (1988) The vestibular nerve of the chinchilla. I. Peripheral innervation patterns in the horizontal and superior semicircular canals. J Neurophysiol 60:167-181.

Fitzpatrick R, McCloskey DI (1994) Proprioceptive, visual and vestibular thresholds for the perception of sway during standing in humans. J Physiol (Lond) 478:173-186.

Gabbiani F (1996) Coding of time varying signals in spike trains of linear and half-wave rectifying neurons. Network 7:61-85.

Goldberg JM (2000) Afferent diversity and the organisation of central vestibular pathways. Exp Brain Res 130:277-297.

Goldberg JM, Fernández C (1971a) Physiology of peripheral neurons innervating semicircular canals of the squirrel monkey. I. Resting discharge and response to constant angular acceleration. J Neurophysiol 34:635-660.

Goldberg JM, Fernandez C (1971b) Physiology of peripheral neurons innervating semicircular canals of the squirrel monkey. II. Response to sinusoidal stimulation and dynamics of peripheral vestibular system. J Neurophysiol 34:661-675.

Goldberg JM, Smith CE, Fernandez C (1984) Relation between discharge regularity and responses to externally applied galvanic currents in vestibular nerve afferents of the squirrel monkey. J Neurophysiol 51:1236-1256.

Green D, Swets J (1966) Signal detection theory and psychophysics. New York: Wiley.

Grossman GE, Leigh RJ, Abel LA, Lanska DJ, Thurston SE (1988) Frequency and velocity of rotational head perturbations during locomotion. Exp Brain Res 70:470-476.

Haque A, Angelaki DE, Dickman JD (2004) Spatial tuning and dynamics of vestibular semicircular afferents in rhesus monkeys. Exp Brain Res 155:81-90.

Hullar TE, Minor LB (1999) High-frequency dynamics of regularly discharging canal afferents provide a linear signal for angular vestibuloocular reflexes. J Neurophysiol 82:2000-2005.

Hullar TE, Della Santina CC, Hirvonen T, Lasker DM, Carey JP, Minor LB (2005) Responses of irregularly discharging chinchilla simicircular canal vestibular-nerve afferents during high-frequency head rotations. J Neurophysiol 93:2777-2786

Hurley KM, Gaboyard S, Zhong M, Price SD, Wooltorton JR, Lysakowski A, Eatock RA (2006) M-like $\mathrm{K}^{+}$currents in type I hair cells and calyx afferent endings of the developing rat utricle. J Neurosci 26:10253-10269.

Huterer M, Cullen KE (2002) Vestibuloocular reflex dynamics during highfrequency and high-acceleration rotations of the head on body in rhesus monkey. J Neurophysiol 88:13-28.

Jarvis MR, Mitra PP (2001) Sampling properties of the spectrum and coherency of sequences of action potentials. Neural Comput 13:717-749.

Johansson RS, Birznieks I (2004) First spikes in ensembles of human tactile afferents code complex spatial fingertip events. Nat Neurosci 7:170-177.

Johnston AR, MacLeod NK, Dutia MB (1994) Ionic conductances contributing to spike repolarisation and after-potentials in rat medial vestibular nucleus neurons. J Physiol (Lond) 481:61-77.

Jones LM, Depireux DA, Simons DJ, Keller A (2004) Robust temporal coding in the trigeminal system. Science 304:1986-1989.

Joseph A, Hyson R (1993) Coincidence detection by binaural neurons in the chick brain stem. J Neurophysiol 69:1197-1211.

Jurgens R, Becker W (2006) Perception of angular displacement without landmarks: evidence for Bayesian fusion of vestibular, optokinetic, podokinesthetic, and cognitive information. Exp Brain Res 174:528-543.

Kreiman G, Krahe R, Metzner W, Koch C, Gabbiani F (2000) Robustness and variability of neuronal coding by amplitude sensitive afferents in the weakly electric fish Eigenmania. J Neurophysiol 84:189-224.

Laurent G, Davidowitz H (1994) Encoding of Olfactory Information with Oscillating Neural Assemblies. Science 265:1872-1875.

Limón A, Pérez C, Vega R, Soto E (2005) $\mathrm{Ca}^{2+}$-Activated $\mathrm{K}^{+}$-currents density is correlated with soma size in rat vestibular-afferent neurons in culture. J Neurophysiol 94:3751-3761.

Lindner B, Chacron MJ, Longtin A (2005) Integrate-and-fire neurons with threshold noise: A tractable model of how interspike interval correlations 
affect neuronal signal transmission. Phys Rev E Stat Nonlin Soft Matter Phys 72:021911.

Lisberger SG, Pavelko TA (1986) Vestibular signals carried by pathways subserving plasticity of the vestibulo-ocular reflex in monkeys. J Neurosci 6:346-354.

Liu YH, Wang XJ (2001) Spike frequency adaptation of a generalized leaky integrate-and-fire neuron. J Comp Neurosci 10:25-45.

Machens CK, Stemmler MB, Prinz P, Krahe R, Ronacher B, Herz AV (2001) Representation of acoustic communication signals by insect auditory neurons. J Neurosci 21:3215-3227.

Meister M (1996) Multineuronal codes in retinal signaling. Proc Natl Acad Sci USA 93:609-614.

Mergner T, Hlavacka F, Schweigart G (1993) Interaction of vestibular and proprioceptive inputs. J Vestib Res 3:41-57.

Minor LB, Goldberg JM (1991) Vestibular-nerve inputs to the vestibuloocular reflex: a functional-ablation study in the squirrel monkey. J Neurosci 11:1636-1648.

Oppenheim AV, Schafer RW (1989) Discrete-time signal processing. Englewood Cliffs, NJ: Prentice Hall.

Ramachandran R, Lisberger SG (2006) Transformation of vestibular signals into motor commands in the vestibuloocular reflex pathways of monkeys. J Neurophysiol 96:1061-1074.

Richmond BJ, Optican LM, Podell M, Spitzer H (1987) Temporal encoding of two-dimensional patterns by single units in primate inferior temporal cortex. I. Response characteristics. J Neurophysiol 57:132-146.

Rieke F, Warland D, de Ruyter van Steveninck RR, Bialek W (1996) Spikes: exploring the neural code. Cambridge, MA: MIT.

Roddey JC, Girish B, Miller JP (2000) Assessing the performance of neural encoding models in the presence of noise. J Comp Neurosci 8:95-112.

Romer A, Parsons TS (1977) The vertebrate body. Philadelphia: Saunders.

Roy JE, Cullen KE (2001) Selective processing of vestibular reafference during self-generated head motion. J Neurosci 21:2131-2142.

Sadeghi SG, Minor LB, Cullen KE (2006) Response of vestibular-nerve afferents to active and passive rotations under normal conditions and following unilateral labyrinthectomy. J Neurophysiol, in press.
Salinas E, Hernandez A, Zainos A, Romo R (2000) Periodicity and firing rate as candidate neural codes for the frequency of vibrotactile stimuli. J Neurosci 20:5503-5515.

Schessel DA (1982) Chemical synaptic transmission between type I vestibular hair cells and the primary afferent nerve chalice: an intracellular study utilizing horseradish peroxidase. $\mathrm{PhD}$ thesis, Albert Einstein College of Medicine, Bronx, NY.

Schessel DA, Ginzberg R, Highstein SM (1991) Morphophysiology of synaptic transmission between type I hair cells and vestibular primary afferents. An intracellular study employing horseradish peroxidase in the lizard, Calotes versicolor. Brain Res 544:1-16.

Smith CE, Goldberg JM (1986) A stochastic afterhyperpolarization model of repetitive activity in vestibular afferents. Biol Cybern 41-51.

Snippe HP, Koenderink JJ (1992) Discrimination thresholds for channelcoded systems. Biol Cybern 66:543-551.

Stein RB, Gossen ER, Jones KE (2005) Neuronal variability: noise or part of the signal. Nat Rev Neurosci 6:4766-4778.

Straka H, Vibert N, Vidal PP, Moore LE, Dutia MB (2005) Intrinsic membrane properties of vertebrate vestibular neurons: function, development, and plasticity. Prog Neurobiol 76:349-392.

Stuart G, Sakmann B (1995) Amplification of EPSPs by axosomatic sodium channels in neocortical pyramidal neurons. Neuron 15:1065-1076.

Sylvestre PA, Cullen KE (1999) Quantitative analysis of abducens neuron discharge dynamics during saccadic and slow eye movements. J Neurophysiol 82:2612-2632.

Tasaki I (1955) New measurements of the capacity and resistance of the myelin sheath and the nodal membrane of the isolated frog nerve fiber. Am J Physiol 181:639-650.

Theunissen F, Miller JP (1995) Temporal encoding in the nervous system: a rigorous definition. J Comput Neurosci 2:149-162.

Theunissen F, Roddey JC, Stufflebeam S, Clague H, Miller JP (1996) Information theoretic analysis of dynamical encoding by four identified interneurons in the cricket cercal system. J Neurophysiol 75: $1345-1364$. 